\title{
Weak lensing observations of potentially X-ray underluminous galaxy clusters
}

\author{
J. P. Dietrich ${ }^{1}$, A. Biviano ${ }^{2}$, P. Popesso ${ }^{3}$, Y.-Y. Zhang ${ }^{4}$, M. Lombardi ${ }^{1}$, and H. Böhringer ${ }^{3}$ \\ 1 ESO, Karl-Schwarzschild-Str. 2, 85748 Garching b. München, Germany \\ e-mail: jdietric@eso.org \\ 2 INAF - Osservatorio Astronomico di Trieste, via G. B. Tiepolo 11, 34143 Trieste, Italy \\ 3 Max-Planck Institut für extraterrestrische Physik, Gießenbachstr., 85748 Garching b. München, Germany \\ 4 Argelander-Institut für Astronomie, Auf dem Hügel 71, 53121 Bonn, Germany \\ Received 27 November 2008 / Accepted 19 March 2009
}

ABSTRACT

\begin{abstract}
Optically selected clusters of galaxies display a relation between their optical mass estimates and their X-ray luminosities $L_{\mathrm{X}}$ that has a large scatter. A substantial fraction of optically selected clusters have $L_{X}$ estimates or upper limits significantly below the values expected from the $L_{X}$-mass relation established for X-ray selected clusters, i.e., these clusters are X-ray underluminous for their mass. We attempt to confirm or falsify the X-ray underluminous nature of two clusters, Abell 315 and Abell 1456, by using weak gravitational lensing as a third and independent measure of the clusters' masses. We obtained optical wide-field imaging data and selected background galaxies using their colors and measured the shear exerted by the tidal field of the foreground galaxy clusters. We then fitted parametrized models to our shear catalogs. After accounting for projections of large-scale structure and halo triaxiality, we find that A 315 is significantly X-ray underluminous for its mass, while no significant lensing signal was detected for A 1456. We re-evaluate earlier kinematic and X-ray analyses of these two clusters and discuss the nature of the X-ray underluminous cluster A 315 and why A 1456 was probably erroneously identified as being X-ray underluminous.
\end{abstract}

Key words. gravitational lensing - galaxies: clusters: individual: A 315 - galaxies: clusters: individual: A 1456

\section{Introduction}

Clusters of galaxies can be detected by various methods: optical (e.g., Gal 2006), X-ray emission (e.g., Böhringer et al. 2001), weak gravitational lensing (e.g., Schneider 1996; Dietrich et al. 2007), and the Sunyaev-Zeldovich effect (e.g., Staniszewski et al. 2008). Differences in the selection method could potentially lead to biases when determining the cluster mass function. Optical selection is generally more affected by projection effects than X-ray selection, although projection effects can be minimized by selecting cluster galaxies on the basis of their colors (e.g., Kim et al. 2002). On the other hand, X-ray selection requires that the intra-cluster gas has been heated to a detectable level, and theoretical predictions show that there is a non-negligible fraction of unvirialized, relatively massive clusters with no X-ray emission (Weinberg \& Kamionkowski 2002). While such systems are mostly expected at high redshifts, they may be present at all epochs.

Most cluster mass functions have been obtained from X-ray selected samples (e.g., Reiprich \& Böhringer 2002). X-ray selection is generally considered to be well understood, almost pure and complete in mass. However, several investigations have questioned the completeness of X-ray selected cluster samples. These investigations have shown that there is a population of optically selected clusters that deviate from the relation between the X-ray luminosity, $L_{\mathrm{X}}$, and virial mass (or an optical mass proxy such as richness and velocity dispersion) established for X-ray selected cluster samples (Bower et al. 1997; McNamara et al. 2001; Donahue et al. 2002; Gilbank et al. 2004; Lubin et al. 2004; Barkhouse et al. 2006; Fang et al. 2007; Popesso et al. 2007a, P07 hereinafter). These clusters are underluminous in X-ray for their masses. P07, in particular, identified X-ray underluminous clusters in the RASS-SDSS survey among Abell clusters, which were therefore named "Abell X-ray Underluminous" (AXU). The virial masses of these AXU clusters were determined from the redshifts of their member galaxies, using data from the Sloan Digital Sky Survey. X-ray luminosities or upper limits to X-ray luminosities were derived from the ROSAT All-Sky Survey.

What is the nature of these AXU clusters? By analyzing the velocity distribution of their members, P07 suggested they are systems in the process of formation. However, it is possible that at least part of the scatter in the $L_{X}$-mass relation is not intrinsic, but originates in erroneous estimates of either the cluster $L_{X}$ or its mass. To investigate this point, we obtained optical wide-field observations of two AXU clusters with the aim of measuring these clusters' masses by means of weak lensing, and deriving a third, independent mass estimate. We describe these data and their reduction in Sect. 2. Section 3 contains the weak lensing analysis, which we compare with revised kinematic and X-ray estimates in Sects. 4 and 5. We revisit the optical cluster luminosity and richness in Sect. 6 and present our conclusions in Sect. 7.

Throughout this paper, we use a standard $\Lambda \mathrm{CDM}$ cosmology with $\Omega_{\mathrm{m}}=0.3, \Omega_{\Lambda}=0.7$, and $H_{0}=70 h \mathrm{~km} \mathrm{~s}^{-1} \mathrm{Mpc}^{-1}$. Confidence intervals correspond to the $68 \%$ confidence level.

\section{Data}

Abell 315, a galaxy cluster at $z=0.174$ (P07), was observed with the Wide-Field Imager (WFI) at the ESO/MPG-2.2 $\mathrm{m}$ telescope in $B-, V$-, and $R$-band. WFI is a focal-reducer type camera 
with a $4 \times 2$ mosaic of $2 \mathrm{k} \times 4 \mathrm{k}$ CCDs with a filling factor of $95.9 \%$ (Baade et al. 1999). Its field-of-view (FOV) is $34^{\prime} \times 33^{\prime}$, resulting in a pixel scale of $0{ }^{\prime} 238$. At the cluster redshift, $1^{\prime}$ corresponds to a physical scale of $177 \mathrm{~h}^{-1} \mathrm{Mpc}$. The observations were carried out in service mode during the nights from Nov. 5 to 12 , 2007 in dark and clear sky conditions. The total exposure times for the three bands were $2880 \mathrm{~s}, 5890 \mathrm{~s}$, and $5500 \mathrm{~s}$, respectively.

Abell 1456, which is at a redshift of $z=0.135$ (P07), was also observed with WFI in the same passbands. For A 1456, 1' corresponds to $144 h^{-1} \mathrm{Mpc}$. These observations were performed during the nights of May 9 and 10, 2008 in dark and clear conditions. The total exposure times for the A 1456 field were $1500 \mathrm{~s}$, $5750 \mathrm{~s}$, and $5500 \mathrm{~s}$ for the $B-, V$-, and $R$-band, respectively.

All data were processed with the GaBoDS/THELI pipeline (Erben et al. 2005). Because no photometric standard stars were observed in the nights that our data were taken, the transformation equations of Koch et al. (2004) were used to calibrate the $V$ - and $R$-band data using SDSS magnitudes of objects in the same fields. The zero points of the $B$-band data were fixed by matching the expected stellar colors of the Pickles (1998) stellar library in a color-color diagram to the observed colors of stars in the fields. The colors of galaxies were corrected for Galactic extinction using the Schlegel et al. (1998) extinction maps. The effective seeing of the coadded images $R$-band images is 0.74 (A 315) and 1'.0 (A 1456).

The observations were divided into observing blocks (OBs) of 5 dithered exposures. Additional offsets between OBs ensured that the sky coverage of the observations was more homogeneous. Every region in the final $V$ - and $R$-band images was covered by at least six exposures. In the shorter $B$-band observations, which used only one $\mathrm{OB}$, every location was covered by at least three exposures. The WFI point-spread-function (PSF) is smooth across chip gaps and has only slow spatial variations, so that the coaddition of dithered exposures does not lead to discontinues in the PSF within the final image.

\subsection{Lensing catalogs}

The Bayesian shape fitting method lensfit ${ }^{1}$ (Miller et al. 2007; Kitching et al. 2008) was used to estimate the shear signal imprinted on the shapes of background galaxies. Stars were preselected in a magnitude-flux-radius diagram with the additional requirement that SExtractor's CLASS_STAR $>0.95$. These stars were modeled with the elliptical Gauss-Laguerre method (Bernstein \& Jarvis 2002; Nakajima \& Bernstein 2007) and a two dimensional 4th order polynomial was fitted to the GaussLaguerre coefficients to obtain a PSF model over the entire WFI field-of-view. Lensfit - in the implementation used here - subdivides the image area into regular grid cells, inside which the PSF is assumed to be constant. Here the side length of the cells was chosen to be 100 pixels or 0'.4. For each cell, an image of the PSF was reconstructed from the shapelet coefficients at the cell's center. These PSF images were then used as a PSF description for lensfit.

The Bayesian prior function proposed by Kitching et al. (2008),

$\mathcal{P}\left(e_{1}, e_{2}\right)=A \cos \left(\frac{|e| \pi}{2}\right) \exp \left[-\left(\frac{2|e|}{\left.B(1+|e|)^{D}\right)}\right)^{C}\right]$

\footnotetext{
${ }^{1}$ If the reader wishes to use the lensfit code, its authors can be contacted at http://www.physics.ox.ac.uk/lensfit/
}

Table 1. Coefficients of the lensfit prior function.

\begin{tabular}{lrrr}
\hline \hline Coeff. & STEP1 & A 315 & A 1456 \\
\hline$B$ & 0.029 & 0.042 & 0.068 \\
$C$ & 0.45 & 0.44 & 0.44 \\
$D$ & 42.7 & 32.6 & 30.5 \\
\hline
\end{tabular}

where $B, C$, and $D$ are free parameters and $|e|=\sqrt{e_{1}^{2}+e_{2}^{2}}$, was used in computing the posterior ellipticity probability distribution. Because of the many degeneracies of this function, a bruteforce minimization was the only viable option in determining its coefficients. The coefficients found here are very similar to those obtained by Kitching et al. (2008) for the STEP1 simulations (Heymans et al. 2006, see Table 1 for a comparison).

Shear estimates for individual galaxies were obtained using Eq. (20) of Kitching et al. (2008). When measuring the shear we must - as in Eq. (1) - assume a prior that contains zero shear because we cannot predict the variation in shear across the FOV. Since after lensing the ellipticity distribution of galaxies is not centered on zero anymore, we need to apply an additional weighting factor to correct for the assumption of zero shear. The shear sensitivity $\partial e_{i} / \partial g_{i}$ describes how the measured ellipticity $e$ of a galaxy depends on the reduced shear $g$. Very low signal-tonoise ratio (SNR) objects have sensitivity values close to zero. Together with high ellipticity measurements, this can lead to unphysically high shear values. Therefore, shear estimates with shear sensitivity of $\partial e_{i} / \partial g_{i}<0.3$ or ellipticity $|e|>1.1$ were rejected. We note that both types of rejection to low SNR objects affected fewer than 50 galaxies, and hence did not bias our lensing estimates.

Because this is the first application of lensfit to real data, we compared the shear estimates obtained in this way with those computed using the Erben et al. (2001) implementation of the Kaiser et al. (1995, KSB) shape estimation algorithm. The STEP1 bias parameters $q$ (non-linear response), $m$ (calibration bias), and $c$ (constant offset) were computed where the lensfit shear estimates were set to $\gamma_{i}^{\text {true }}$. We found that $q$ and $c$ are consistent with zero and that $\bar{m}=0.152 \pm 0.029$. This is consistent with the bias parameter values of the MH implementation of KSB in Heymans et al. (2006), which is, save a few details, identical to the KSB implementation used here. This result suggests that, apart from errors in the PSF model common to both methods, the lensfit shear estimates are very close to the true shear values.

Background galaxies were selected based on their magnitude and colors. Galaxies with $R>23$ mag were considered to be background galaxies and were included in the catalog irrespective of their colors, while brighter galaxies were rejected if their colors matched the colors of cluster galaxies, $0.53<(B-V)<$ 1.38 and $-0.25<1.7 \times(V-R)-(B-V)<0.3$. Finally, only galaxies manually preselected in a magnitude-flux-radius diagram were kept if their flux radius was $<1$ ". 3 . The effectiveness of this color selection was tested by plotting the number density of galaxies in radial bins from the cluster centers. A small excess of galaxies was found only for A 315 within a clustercentric distance of 1'.2; beyond this radius, the number density remained constant, which is indicative of no contamination of the background catalogs by cluster galaxies.

After these selections, 9505 galaxies with shear estimates remained in the lensing catalog of A 315, and 8030 galaxies were left in the A 1456 catalog, corresponding to respective number densities of $8.6 \mathrm{arcmin}^{-2}$ and $7.5 \mathrm{arcmin}^{-2}$, where areas masked 

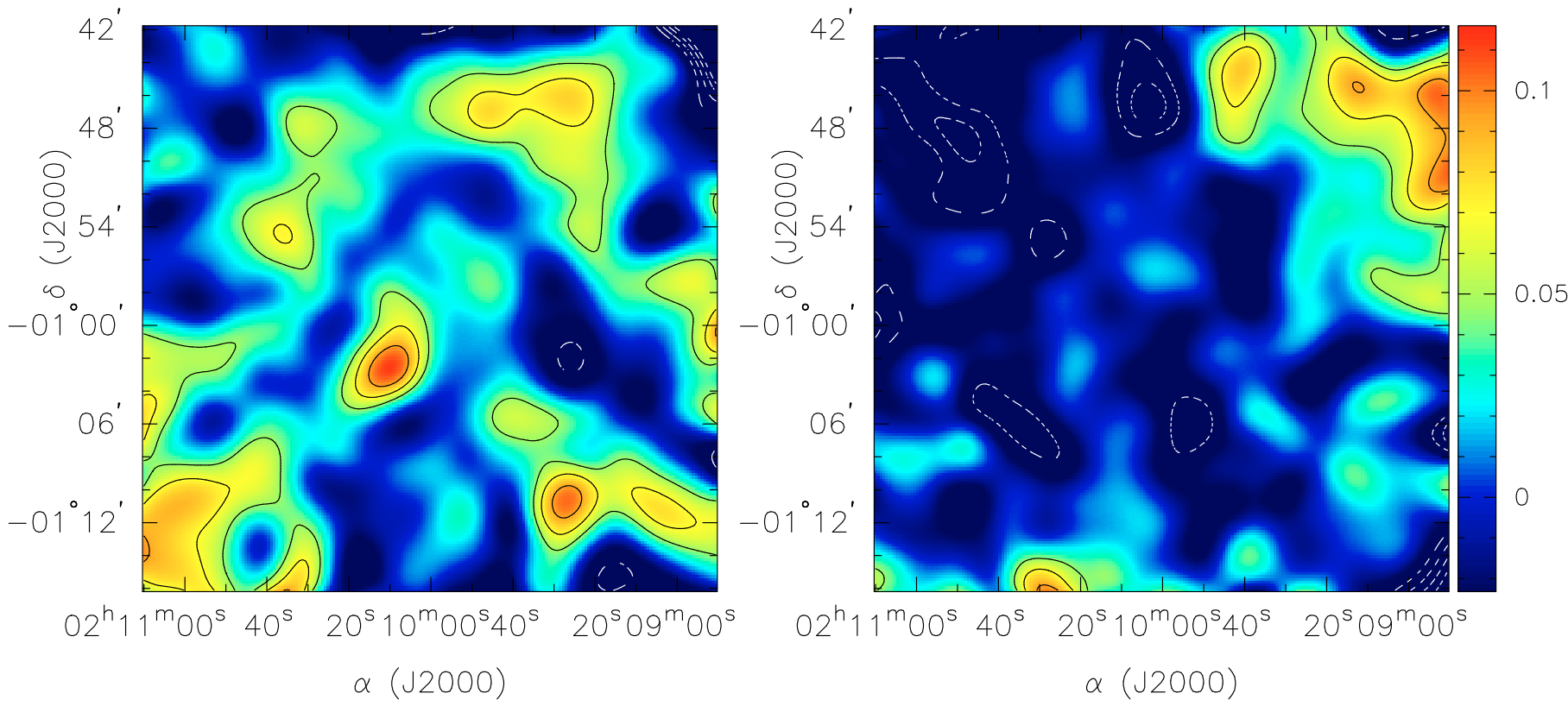

Fig. 1. Left: mass reconstruction of the A 315 field. The surface mass density contours start at at 0.0464 (corresponding to $1.6 \times 10^{14} h M_{\odot} \mathrm{Mpc}^{-2}$ or $2 \sigma$ above the mean surface mass density at the edge of the field), increasing in steps of $1 \sigma$. Dashed contours are at the same negative levels. Right: B-mode reconstruction of the A 315 field. Contours are at the same levels as in the left panel.
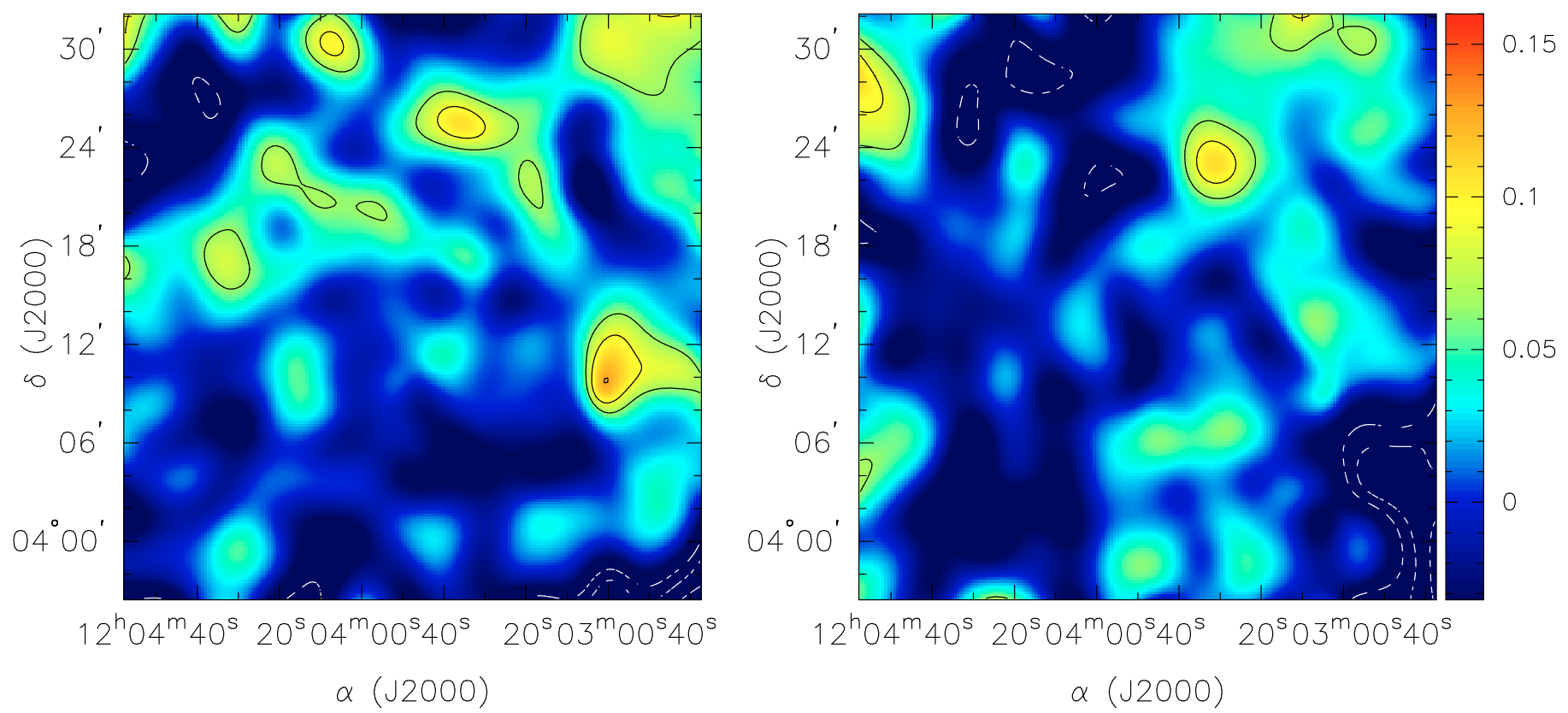

Fig. 2. Same as Fig. 1 for the A 1456 field. The $1 \sigma$ level is at 0.032 (corresponding to $1.25 \times 10^{14} h M_{\odot} \mathrm{Mpc}^{-2}$ ). The color bar on the right runs from $-1 \sigma$ to $5 \sigma$ in both figures.

due to the occurrence of bright stars, reflection rings, and diffraction spikes were excluded.

\section{Weak lensing mass estimates}

Based on the lensing catalogs described in the previous section, surface mass density maps were computed using the finite field inversion method of Seitz \& Schneider (2001) with a smoothing scale of $2^{\prime}$. These maps are presented in the left panels of Figs. 1 and 2. The right panels of these figures are B-mode maps obtained from shear catalogs with galaxies rotated by $45^{\circ}$ to cancel any true shear signal, and is a test of systematic residuals in the PSF correction. The flatness of the B-mode maps suggests that the PSF models used in creating the lensing catalogs are sufficiently accurate for obtaining cluster mass estimates. The noise levels of the reconstructions were estimated from the variance of 100 maps with randomized galaxy orientations.

At a confidence level just under $5 \sigma$, A 315 is clearly detected a little SE of the image center. No lensing signal is visible at the position of A 1456 at the center of the image. The strong peak at the right of the image is probably caused by the reflection ring of a bright star on which it is centered. However, this peak is also only 45" away from the cluster candidate NSC J120257+040951 (Gal et al. 2003).

The weak lensing peak of A 315 is centered on a galaxy concentration whose brightest galaxy is 2 .' 2 from the cluster position 
of Abell et al. (1989) and 4.3 from the cluster center adopted in the X-ray and kinematic analysis of P07. Both the position of P07 and the brightest cluster galaxy (BCG) identified here (02:10:06.46, -01:01:56.46), are compatible with the positional uncertainties of Abell et al. (1989) but are mutually incompatible. Because the $\mathrm{P} 07$ position of A 315 was based on a very weak $\mathrm{X}$-ray signal, the BCG position was adopted as the cluster center for the analysis presented here, a choice that we further justify in Sect. 4, where the kinematic analysis of both clusters is reexamined. For A 1456 the weak lensing analysis was done taking the BCG identified in Sect. 4 (12:03:48.71, +04:20:43.46), located 5.75 from the X-ray center of P07, to be the center.

Converting the dimensionless lensing quantities into physical mass densities requires knowledge of the source redshift distribution or at least of the average source redshift. To determine the latter quantity, galaxies were randomly drawn from the photometric redshift catalog of Ilbert et al. (2006) to match the magnitude distribution of our lensing catalogs, using the color transformations of Blanton \& Roweis (2007). The average redshifts of the shear catalogs are $\bar{z}_{\mathrm{A} 315}=1.07$ and $\bar{z}_{\mathrm{A} 1456}=1.03$. While it is formally possible to estimate errors in these mean redshifts by bootstrap resampling from the CFHTLS catalogs, the true redshift error will be dominated by cosmic variance, which we do not explicitly calculate here. We note that for the two lowredshift clusters under investigation, a redshift error as high as 0.2 would result in a mass error of less than $5 \%$ and even in this extreme case would be an insignificant contribution to the total error budget of the lensing mass estimates.

To obtain mass estimates, parametrized models - singular isothermal spheres (SIS) and NFW profiles - were fitted using the maximum likelihood method of Schneider et al. (2000). The best-fit SIS model for A 315 has a velocity dispersion of $\sigma_{\text {SIS }}=747_{-82}^{+85} \mathrm{~km} \mathrm{~s}^{-1}$. The best-fit NFW model for A 315 has $M_{200}=2.96_{-0.75}^{+1.15} \times 10^{14} h^{-1} M_{\odot}$. The minimization was carried out with a Downhill Simplex method (e.g., Press et al. 1992) in which at every vertex the concentration parameter was fixed to the prescription of Dolag et al. (2004). Attempts to fit parametrized models to A 1456 returned only marginally significant results. The best-fit SIS has $\sigma_{\text {SIS }}=418_{-257}^{+150} \mathrm{~km} \mathrm{~s}^{-1}$, and the NFW model has $M_{200}=6.3_{-5.1}^{+7.0} \times 10^{13} h^{-1} M_{\odot}$. All model fits were centered on the BCG.

Hoekstra (2001, 2003) and Dodelson (2004) studied the influence of uncorrelated large-scale structure (LSS) on cluster mass estimates. Dodelson (2004) found that LSS projections can increase the error in $M_{200}$ by as much as $75 \%$ when the concentration parameter $c$ and $M_{200}$ are fitted simultaneously. Much of this additional error comes from the stretching of the error ellipsis along the $c$-axis, and it was estimated from Fig. 3 of Dodelson (2004) that in the case of fixed $c$, as used in this work, the additional error is at most $50 \%$.

Corless \& King (2007) investigated the influence of halo triaxiality on weak-lensing mass estimates and found that very oblate or prolate halos with axis ratio 1:3 decrease or increase weak-lensing mass estimates by as much as $40 \%$, depending on their orientation with respect to the line of sight. These are, however, extreme cases, which occur in less than $1 \%$ of all halos (Kasun \& Evrard 2005) and a realistic estimation of the contribution to the total error budget must take the halo-shape distribution into account. Shaw et al. (2006), for example, found that prolate halos, i.e., those halos that according to Corless \& King (2007) lead to higher additional errors in weak lensing measurements, are much more common than oblate halos. Kasun $\&$ Evrard (2005) presented a fitting function for the minor-axis

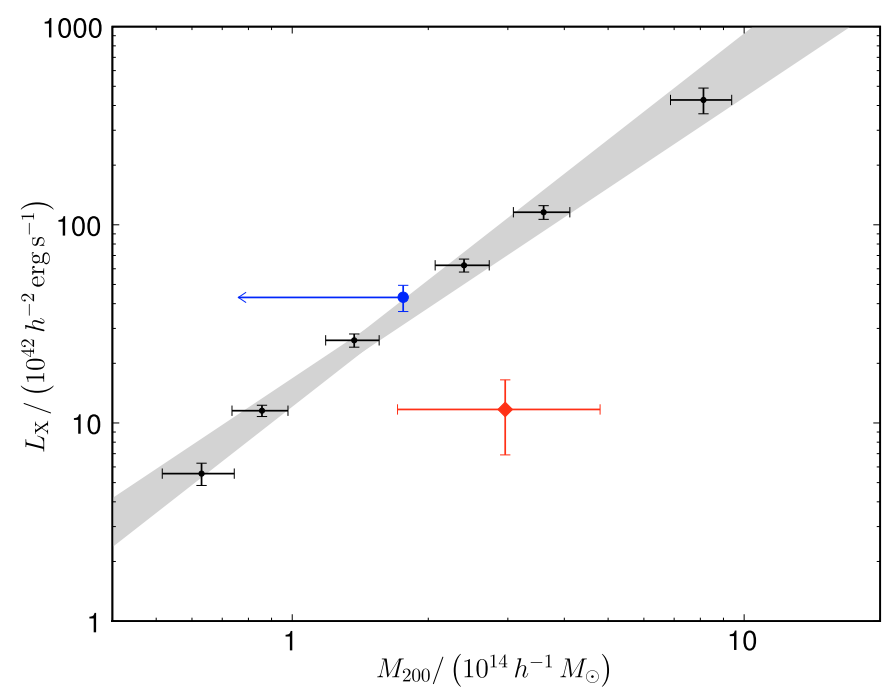

Fig. 3. The clusters A 315 (red diamond) and A 1456 (blue circle) in comparison with the $L_{X}-M_{200}$ relation of Rykoff et al. (2008, black dots and shaded area). The error bars for the mass of A 315 are the sum of random and systematic errors. For A1456, we use the $1 \sigma$ random plus systematic upper limit to the mass estimate, since the cluster is undetected in the weak lensing analysis.

as a function of mass and redshift. For a halo at the redshift and mass of A 315 the expected $c / a=0.63$. From Figs. 4 and 5 of Corless \& King (2007), we found that in the extreme cases of the major (minor) axis being aligned with the line of sight, the weak-lensing mass for this axis ratio will be overestimated (underestimated) by $16 \%(10 \%)$. Because the results of Corless \& King (2007) cannot be convolved with the full halo-shape distribution, we adopt this combination of mean axis ratio and extreme alignment as additional error from halo triaxiality.

Adding the systematic errors of LSS projections and halo triaxiality leads to total errors that are significantly higher than those obtained from the model fits alone. For A $315 M_{200}=$ $\left(2.96_{-0.75-0.50}^{+1.15+0.69}\right) \times 10^{14} h^{-1} M_{\odot}$ was found, where the first error is random and the second error is sytematic. The marginal detection of A 1456 turns into an upper limit to the mass if projection effects of LSS are taken into account, $M_{200}=\left(6.3_{-5.1-3.4}^{+7.0+4.2}\right) \times$ $10^{13} h^{-1} M_{\odot}$.

\section{Kinematical mass estimates}

The lensing analysis in the previous section established that the position of A 315 is differs significantly from the one used in the kinematical analysis of P07. Because the choice of centroid position affects the selection of cluster members, the kinematic analysis of SDSS DR5 data (Adelman-McCarthy et al. 2007) was repeated using the BCG as the cluster center. This analysis follows the steps of Biviano et al. (2006), as briefly summarized here:

1. a peak in the redshift distribution is selected;

2. interlopers are rejected by analyzing the projected phasespace diagram, i.e., velocities versus clustercentric distances;

3 . the virial mass and velocity dispersion are computed within a given aperture;

4. the computed mass and velocity dispersion are translated into the mass within $r_{200}$. 


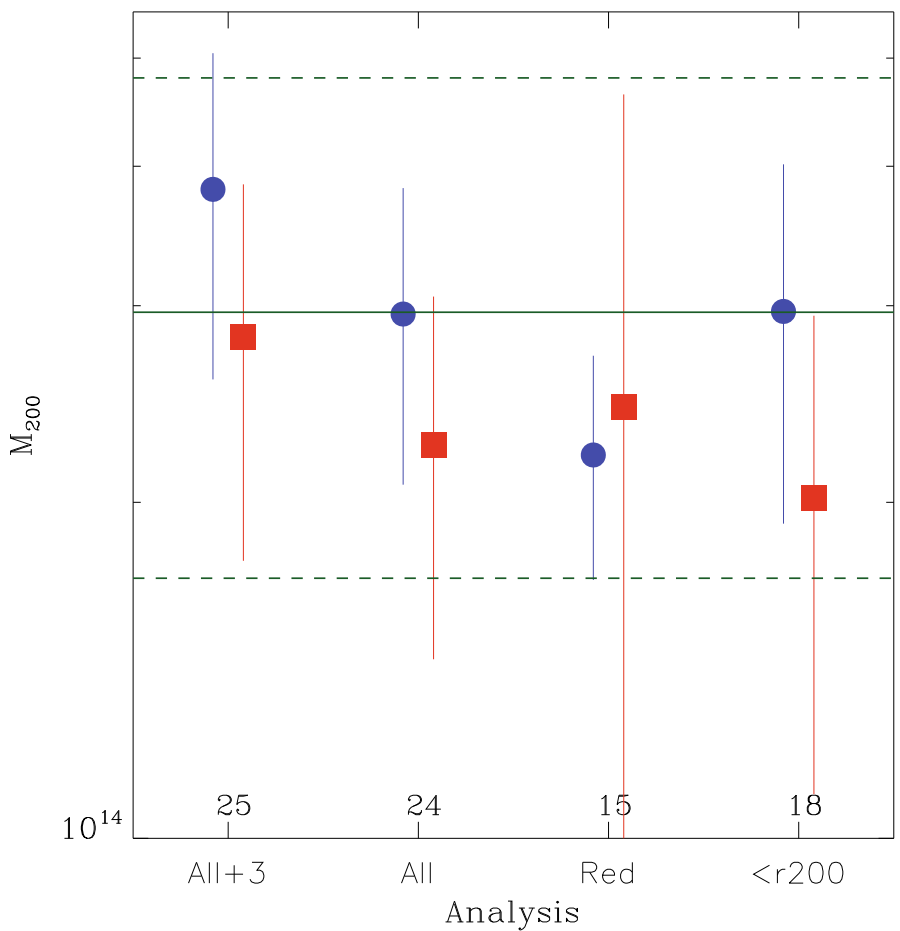

Fig. 4. Comparison of kinematic and weak-lensing mass estimates of A 315. The filled (blue) circles are virial mass estimates; the filled (red) squares are mass estimates obtained from the velocity dispersion. Shown are the estimates for the samples of (1) all galaxies plus the 3 outliers (see text for details); (2) all galaxies; (3) only red-sequence galaxies; and (4) galaxies within $r_{200}$. The numbers on the $x$-axis indicate the sample sizes. The solid (green) line shows the weak-lensing mass estimate with error bars (dashed lines) for comparison.

The mass estimates are corrected for the surface-pressure term (The \& White 1986). In the following, we describe the influence of these steps on the kinematical mass estimates of A 315.

Step 1 requires the choice of an aperture centered on the adopted center position to select all galaxies within this aperture. Weighted gap (Beers et al. 1990) and the density gap (Adami et al. 1998) estimators with a gap value of 4 were chosen to select the peak position. We note that our mass estimates are insensitive to the choice of the gapper method and of the gap value. However, the mass value depends on the initial aperture for the search of the peak. Adopting a radius of $2 h^{-1} \mathrm{Mpc}$ instead of $1 h^{-1}$ Mpc causes the mass estimate of A 315 to increase by $31 \%$. This is because of the inclusion of 3 galaxies in the data-set that are excluded when an aperture of $1 h^{-1} \mathrm{Mpc}$ is used. With the exception of these 3 galaxies, the identification of interlopers (step 2) is quite robust, with little room for a different selection.

The mass estimate in step 3 may be computed within an aperture that differs from the one used in step 1 by being larger. Here one Abell radius, $2.15 h^{-1} \mathrm{Mpc}$, was adopted. The mass within this radius is used to obtain a first estimate of $r_{200}$, and then to interpolate from the Abell radius to $r_{200}$ using an NFW profile and obtain $M_{200}$ (step 4). Alternatively, instead of using the Abell radius, the mass within the aperture corresponding to $r_{200}$ can be computed. Since the $r_{200}$ estimate will change as a result of the aperture selection, this is done iteratively. The difference between the mass estimates of both methods is negligible.

Rather than using all cluster galaxies, one can use only those on the red sequence. In this case, we found that the mass estimate decreases by $25 \%$, if the mass estimated is obtained within an aperture of $2.15 h^{-1} \mathrm{Mpc}$.

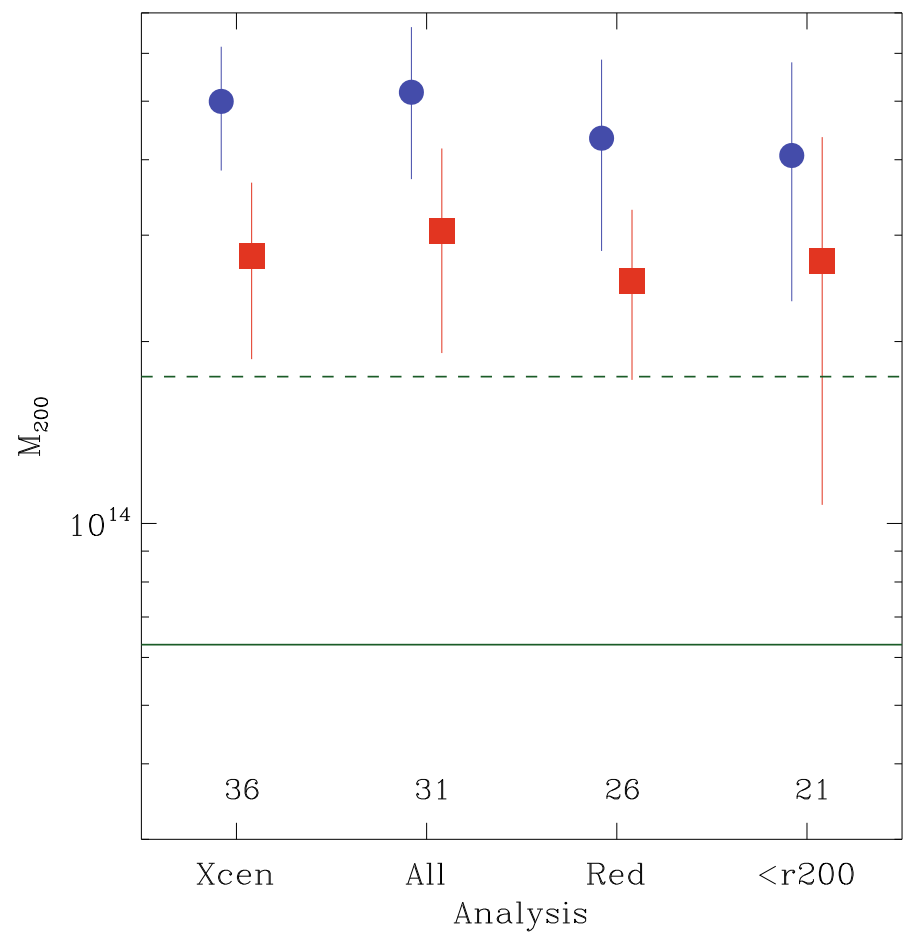

Fig. 5. Same as Fig. 4 but for A 1456. The leftmost point is for the analysis performed at the X-ray cluster position. All other points were computed with the aperture centered on the BCG.

Two mass estimates are reported at the end of each analysis. One comes from the virial analysis and the other uses the velocity dispersion as a proxy. It is known from numerical simulations (Biviano et al. 2006) that the former is generally an overestimate and the latter an underestimate, so that the two values should bracket the real one.

Mass errors were computed with the Jackknife technique. They range from $23 \%$ to $35 \%$ for the virial mass, but are much higher for the mass derived from the velocity dispersion (from $36 \%$ to $90 \%$ ). The various mass estimates of A 315 are displayed in Fig. 4. All mass estimates reported here are lower than the value of $(6.61 \pm 2.71) \times 10^{14} h^{-1} M_{\odot}$ reported by P07. The difference is due to the different central position adopted here, the BCG rather than the X-ray center. This centroid choice also decreases the systematic error estimates because the main peak seems to be more reliably centered.

We consider the range in the estimates obtained by changing the parameters of the analysis to be systematic error, and the average jackknife errors of these estimates as random error. The cluster mass is now estimated to be $\left(2.7_{-0.7}^{+1.1} \pm 1.0\right) \times 10^{14} h^{-1} M_{\odot}$, where the first error is the random error and the second one is systematic.

According to a Dressler \& Shectman (1988) test, there is no evidence of subclustering, but this test is not very reliable for samples of redshift for fewer than 50 galaxies; there is also no evidence of a gradient in the $R-v$ plane.

The kinematic analysis was also repeated for A 1456, where it was found that, compared to the A 315 case, the mass estimates obtained for A 1456 depend very little on details of the membership selection for galaxies in the cluster field. Variations in (1) the choice of the cluster center, (2) the selection of all vs. red-sequence galaxies only, and (3) the choice of the limiting aperture for the analysis, lead to variations in the virial mass estimates in the range $4.1-5.2 \times 10^{14} h^{-1} M_{\odot}$, and in the velocity dispersion mass estimates in the range $2.5-3.1 \times 10^{14} h^{-1} M_{\odot}$. 


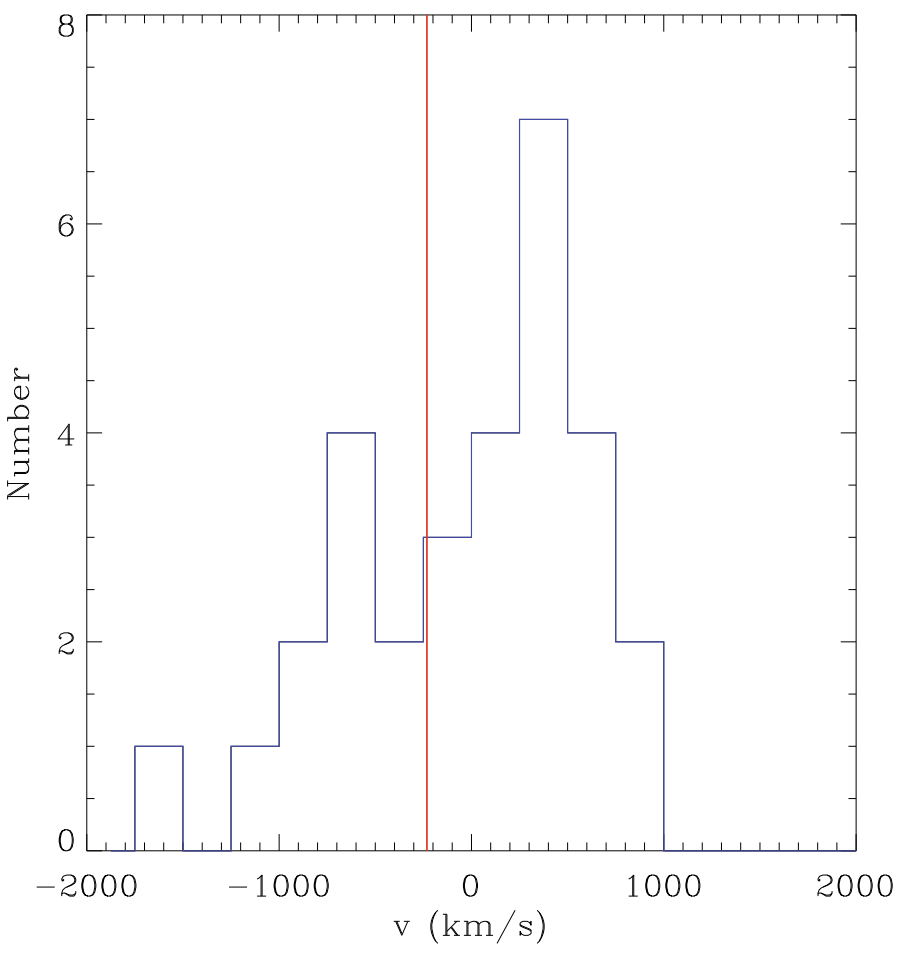

Fig. 6. Velocity histogram of A 1456. Velocities are given with respect to the mean velocity. The vertical red line denotes the velocity of the BCG.

These values agree with those listed in $\mathrm{P} 07$ but are significantly above the mass limit of $1.75 \times 10^{14} h^{-1} M_{\odot}$ derived from the weak-lensing analysis. A comparison of the weak lensing and the different kinematical mass estimates is shown in Fig. 5.

There is only marginal indication of subclustering in the A 1456 data. There is no gradient in the radius-velocity plot, but a marginally significant result of the Dressler \& Shectman test for the red-sequence galaxies $(96 \%$ probability of subclustering). Moreover, the velocity histogram for all galaxies within an Abell radius $\left(2.15 h^{-1} \mathrm{Mpc}\right)$ is not symmetric, with a skewness of -0.62 that is marginally significant (90-95\%). Finally, the BCG is offset from the mean velocity by $-231 \mathrm{~km} \mathrm{~s}^{-1}$ but the difference is within $1.6 \sigma$ of the mean velocity. The velocity histogram is shown in Fig. 6 .

\section{X-ray data analysis}

Based on the weak-lensing and revised kinematical mass estimates, we also reanalyzed the X-ray data of A 315 and A 1456 in the RASS. The cluster temperature was calculated from the cluster mass at $r_{500}$ using the mass-temperature relation in Zhang et al. (2008, Table 3).

The X-ray luminosity, $L_{X}$, depends on the square of the electron density, $n_{\mathrm{e}}^{2}$, and the emission coefficient, $\Lambda, L_{\mathrm{X}} \propto$ $\int n_{\mathrm{e}}^{2} \Lambda(T, Z) \mathrm{d} V$. The luminosity can be derived from the X-ray surface brightness if the emission coefficient is known (e.g., Zhang et al. 2005). The emission coefficient is a function of gas temperature and metallicity. It can be derived in XSPEC using a spectral model for the cluster. Here we used a combined model of "wabs $\times$ raymond" (Morrison \& McCammon 1983; Raymond $\&$ Smith 1977). The "raymond" model describes the cluster thermal component including parameters of the gas temperature, $T$, gas metallicity (assuming $Z=0.4 Z_{\odot}$ ), and cluster redshift. The "wabs" model is set to the hydrogen column density from the
LAB survey (Hartmann \& Burton 1997; Kalberla et al. 2005), $2.5 \times 10^{20} \mathrm{~cm}^{2}$ for A 315 and $1.65 \times 10^{20} \mathrm{~cm}^{2}$ for A 1456 .

Both A 315 (ID: 931706) and A 1456 (ID: 931633) were observed with the ROSAT PSPC in the RASS. One can subtract the background from the X-ray image to obtain the cluster image. The cluster surface brightness is the X-ray image divided by the exposure map, which was used within the virial radius to determine the X-ray luminosity.

The cluster temperature of A 315 derived from the weak lensing mass is $3.33 \mathrm{keV}$. We used the BCG position to determine the X-ray luminosity. An attempt to determine an X-ray position for A 315 resulted in a non-significant detection at (02:10:07.263,-00:59:49.98). The X-ray luminosity changes by less than $1 \%$ if the cluster center is shifted to this location. The derived cluster X-ray luminosity is $1.17 \pm 0.48 \times 10^{43} h^{-2} \mathrm{erg} \mathrm{s}^{-1}$ in the $0.1-2.4 \mathrm{keV}$ band. The X-ray luminosity of the BCG (02:10:06.458,-01:01:56.46) is below the background level. We therefore used the background level to estimate an upper limit to the X-ray luminosity of the BCG inside a radius of 1.5. This is $L_{\mathrm{X}}<4.58 \times 10^{42} \mathrm{~h}^{-2} \mathrm{erg} \mathrm{s}^{-1}$ in the $0.1-2.4 \mathrm{keV}$ band.

The cluster temperature derived from the kinematical mass $\left(M_{200}=2.7 \times 10^{14} h^{-1} M_{\odot}\right)$ is $3.14 \mathrm{keV}$. The derived cluster $\mathrm{X}$-ray luminosity is $1.18 \pm 0.48 \times 10^{43} \mathrm{~h}^{-2} \mathrm{erg} \mathrm{s}^{-1}$ in the $0.1-$ $2.4 \mathrm{keV}$ band. The X-ray luminosity varies by not more than $1 \%$ for the different cluster masses derived in Sects. 3 and 4. We note that all X-ray luminosities given here for A 315 are derived from very marginal detections. The dominant error in these luminosities is probably the background subtraction and the true luminosity could be substantially lower.

The cluster temperature of A 1456 derived from the weaklensing mass $\left(M_{200}=0.63_{-0.80}^{+1.13} \times 10^{14} h^{-1} M_{\odot}\right)$ is $1.24 \mathrm{keV}$. We used the X-ray cluster center $(12: 03: 45.7,+04: 15: 00)$ in our analysis. The $1 \sigma$ error of the X-ray center position is $3^{\prime}$, i.e., about $2 \sigma$ away from the BCG. The X-ray emission at the BCG position is not significantly above the background.

The derived cluster $\mathrm{X}$-ray luminosity is $2.88 \pm 0.51 \times$ $10^{43} h^{-2} \mathrm{erg} \mathrm{s}^{-1}$ in the $0.1-2.4 \mathrm{keV}$ band. The X-ray luminosity at the BCG position $(12: 03: 48.700,+04: 20: 44.00)$ within a radius of $1.5^{\prime}$ is $0.91 \pm 0.91 \times 10^{42} h^{-2} \mathrm{erg} \mathrm{s}^{-1}$ in the $0.1-2.4 \mathrm{keV}$ band, which is the sum of the cluster luminosity in this region and the BCG luminosity.

Because the weak-lensing mass estimate is compatible with no mass being present at the cluster location, we also used the upper limit to the weak-lensing mass $\left(M_{200}=1.75 \times\right.$ $10^{14} h^{-1} M_{\odot}$ ) to estimate an upper limit of the X-ray luminosity. This gives a cluster temperature of $2.37 \mathrm{keV}$. The derived cluster X-ray luminosity is $4.43 \pm 0.65 \times 10^{43} h^{-2} \mathrm{erg} \mathrm{s}^{-1}$ in the $0.1-2.4 \mathrm{keV}$ band. It is this luminosity that we plot in Fig. 3.

The cluster temperature corresponding to the upper value of the kinematical mass $\left(M_{200}=5.18 \times 10^{14} h^{-1} M_{\odot}\right)$ is $4.67 \mathrm{keV}$. The derived cluster X-ray luminosity is $4.32 \pm 0.66 \times$ $10^{43} h^{-2} \mathrm{erg} \mathrm{s}^{-1}$ in the $0.1-2.4 \mathrm{keV}$ band.

\section{Optical cluster luminosity and richness}

After establishing that A 315 indeed seems to be X-ray underluminous for its mass and that A 1456 is is in agreement with the normal $L_{X}-M_{200}$ relation but has an unusually high kinematical mass estimate, we now examine whether the optical properties of these clusters are in any way exceptional. Specifically, we determined their cluster luminosities and richnesses and compared them to the full RASS-SDSS cluster catalog.

The total optical luminosity of a cluster has to be computed after the subtraction of the foreground and background galaxy 


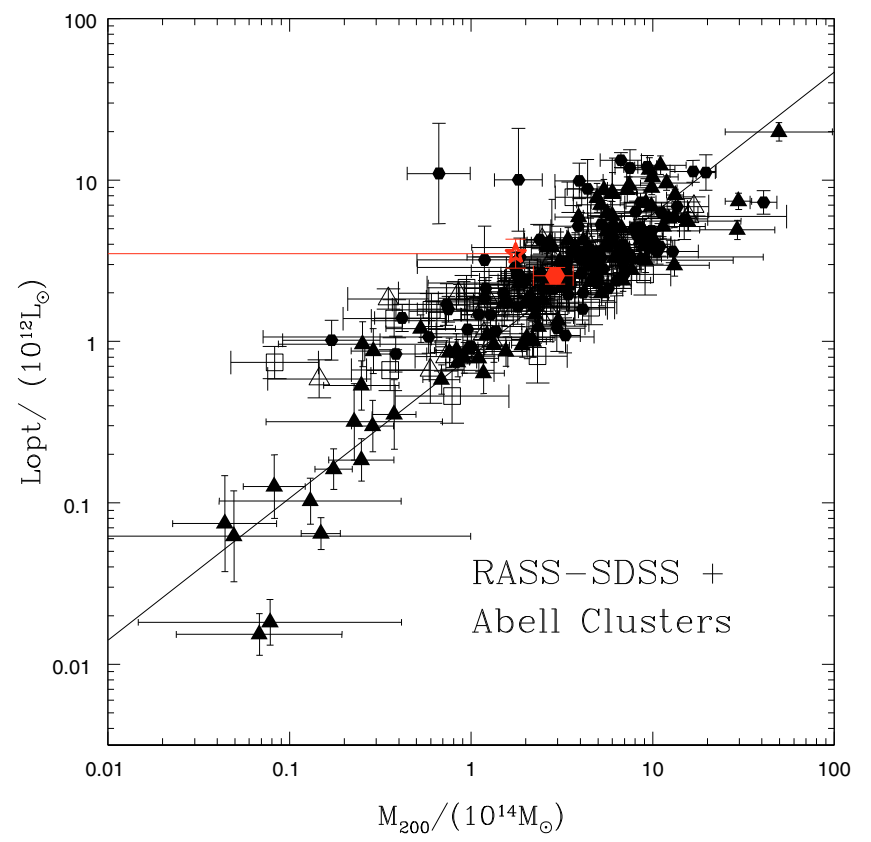

Fig. 7. Location of Abell 315 and Abell 1456 in the $L_{\mathrm{op}}-M_{200}$ relation. The optical luminosity is calculated inside $r_{200}$ and is corrected for contamination due to projection effects. The red filled hexagon refers to Abell 315 and the red star is the upper limit for Abell 1456. The empty black squares and the filled points are the X-ray and optically selected clusters, respectively, used in Popesso et al. (2007b). The solid and dashed line are the best-fit lines obtained in Popesso et al. (2007b).

contamination. We considered two different approaches to the statistical subtraction of the galaxy background. We computed the local background number counts in an annulus around the cluster and a global background number counts from the mean of the magnitude number counts determined in five different SDSS sky regions, randomly chosen, each with an area of $30 \mathrm{deg}^{2}$. In our analysis, we show the results obtained using the optical luminosity estimated with the second method. The optical luminosity is then computed following the prescription of Popesso et al. (2004). The reader is referred to that paper for a detailed discussion of the comparison between optical luminosities calculated with different methods. To avoid selection effects due to the slightly different redshifts of the clusters, the optical luminosity was calculated in the same absolute magnitude range for all the clusters. We used an absolute magnitude cut of $M_{r} \leq-20$, which allowed us to sample the cluster luminosity function down to $M^{*}+2$ (Popesso et al. 2005).

The cluster richness $N_{\text {gal }}$ was calculated by summing the background-subtracted cluster number counts used to calculate $L_{\mathrm{op}}$. This estimate was corrected for projection effects in the same way as we did for $L_{\mathrm{op}}$, according to the prescription given in Popesso et al. (2007b).

The cluster optical luminosity and richness were calculated within a physical aperture of $r_{200}$. According to the weak-lensing results, we used $r_{200}=1.30 h^{-1} \mathrm{Mpc}$ for A 315. For Abell 1456 we used the upper limit to the virial radius, $r_{200}=1.10 h^{-1} \mathrm{Mpc}$, corresponding to the weak-lensing limit of $1.75 \times 10^{14} M_{\odot}$.

Figures 7-9 show the location of A 315 (red filled hexagon) and A 1456 (red star) in the $L_{\mathrm{opt}}-M_{200}, M / L-M_{200}$ and $N_{\text {gal }}-M_{200}$ relations, respectively, obtained by Popesso et al. (2007b).

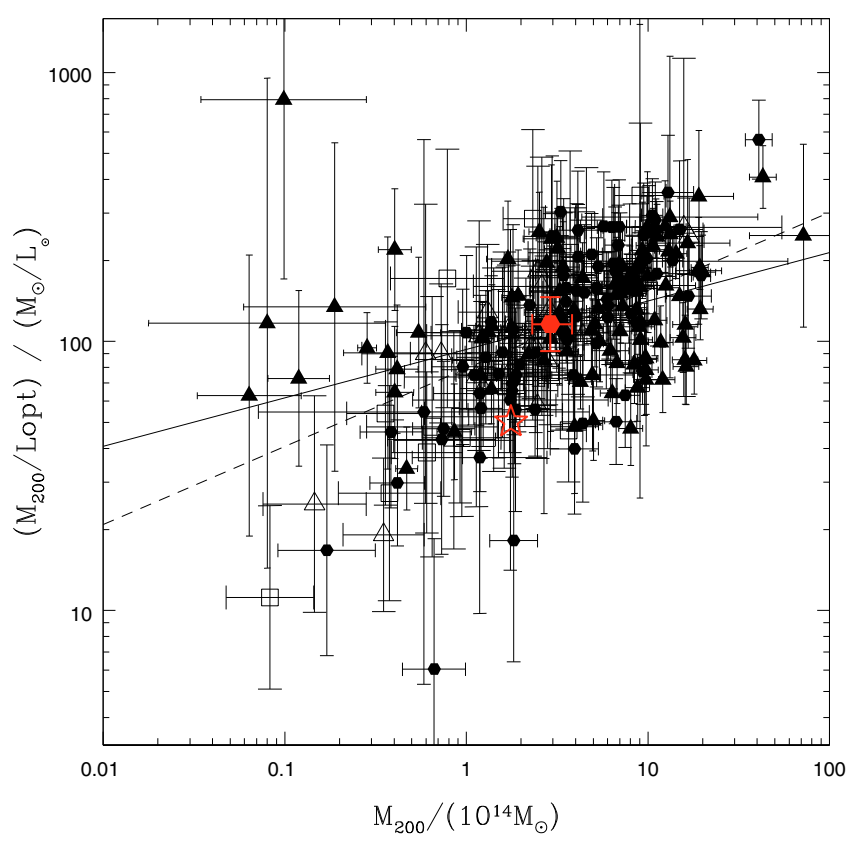

Fig. 8. Location of Abell 315 and Abell 1456 in the $M_{200} / L_{\mathrm{op}}-M_{200}$ relation. The symbols are the same as in Fig. 7

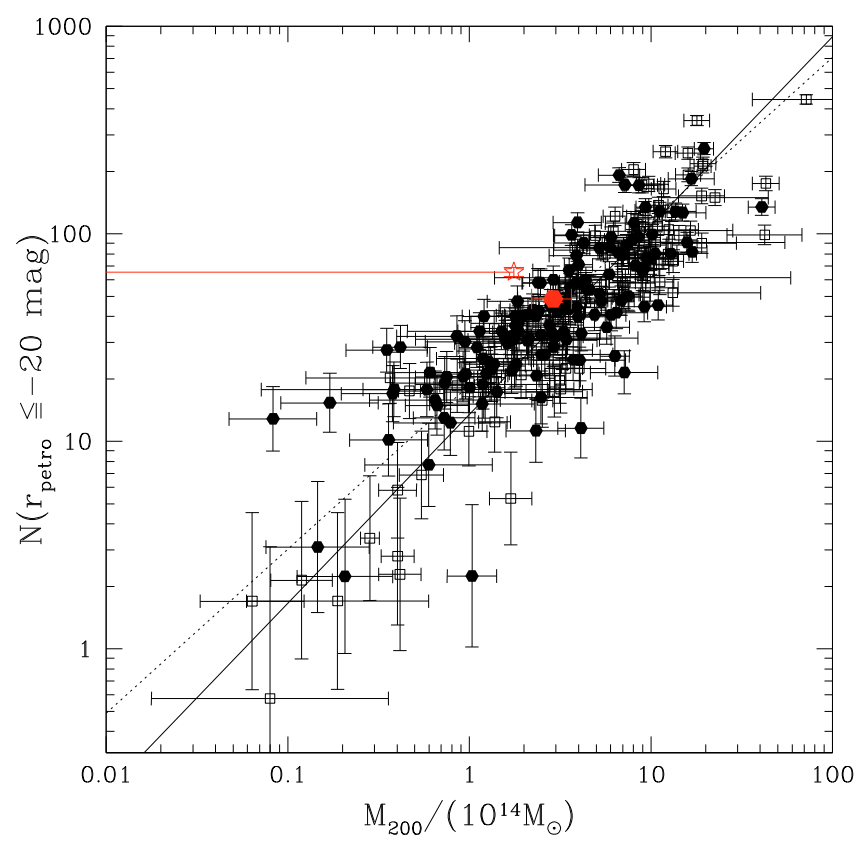

Fig. 9. Location of Abell 315 and Abell 1456 in the $N_{\text {gal }}-M_{200}$ relation. The symbols are the same as in Fig. 7.

\section{Discussion and conclusions}

We have studied two clusters of galaxies that were previously reported to be X-ray underluminous on the basis of their their kinematically derived masses. To either confirm or falsify the $\mathrm{X}$-ray underluminous nature of these clusters, we obtained deep multi-color optical imaging to measure the cluster masses using the gravitational lens effect.

Our lensing analysis of Abell 315 confirmed that this system is a massive cluster, with a lensing mass estimate of $M_{200}=$ $\left(2.96_{-0.75-0.50}^{+1.15+0.69}\right) \times 10^{14} h^{-1} M_{\odot}$. This is substantially lower than the 
kinematical mass estimate of $M_{200}=(6.61 \pm 2.71) \times 10^{14} h^{-1} M_{\odot}$ reported by $\mathrm{P} 07$. We could explain this discrepancy by the choice of cluster center in P07. Shifting the center in the kinematical analysis to that of the BCG in this work leads to a revised kinematical mass estimate of $M_{200}=\left(2.7_{-0.7}^{+1.1} \pm 1.0\right) \times 10^{14} h^{-1} M_{\odot}$, which is in excellent agreement with the weak-lensing mass estimate.

To reliably establish the X-ray underluminous nature of A 315, we also reanalyzed the available X-ray data in the RASS. The X-ray luminosity $L_{\mathrm{X}}=(1.18 \pm 0.48) \times 10^{43} h^{-2} \mathrm{erg} \mathrm{s}^{-1} \mathrm{de}-$ termined in this work is somewhat higher than the value given by Popesso et al. (2007a) but is still significantly below the $L_{X}-$ $M_{200}$ relation of Rykoff et al. (2008).

The lensing analysis of Abell 1456 field did not show a massive cluster. Our attempts to fit parametric models centered on the BCG position returned only marginally significant results, as would be expected from random fluctuations due to shape noise and the projections of LSS. We therefore interpret the lensing measurement as an upper limit to the mass of a cluster at this position and redshift, $M_{200}<1.8 \times 10^{14} h^{-1} M_{\odot}$. This is significantly lower than the kinematic mass estimate that we find for A 1456, which seems to be very robust.

A possible explanation of the much lower mass determined by weak lensing could be that A 1456 is a bimodal cluster that the kinematic analysis is unable to model accurately, given that indications of subclustering are only marginal. If the cluster is indeed bimodal, as suggested by the velocity histogram, the position of the BCG in-between the two velocity peaks suggests that it has been displaced from one of the two peaks by an interaction. Hence this would imply that the kinematic mass estimate is incorrect.

The X-ray analysis of A 1456 shows a much clearer detection than in the case of A 315. By using the upper mass limit derived from the weak lensing analysis to determine to which radius the X-ray luminosity is measured, we can place A 1456 right on the $L_{X}-M_{200}$ relation of Rykoff et al. (2008), and the X-ray luminosity based on the kinematic mass places A 1456 slightly above the $L_{X}-M_{200}$ relation.

In summary, our results show that A 315 is indeed significantly below the $L_{X}-M_{200}$ relation, confirming that it is underluminous compared to X-ray selected galaxy clusters of the same size. We note that the error in the X-ray luminosity is probably dominated by the uncertainty in the background model and higher than the one quoted in Sect. 5. We could not confirm that A 1456 is an AXU cluster. The absence of a significant lensing signal in combination with the marginal indication of bimodality in the velocity histogram make the explanation that this is an unrelaxed cluster merger more likely.

The revised optical luminosity and richness of both clusters are unremarkable. The optical properties give no indication that A 315 is either X-ray underluminous or dynamically unrelaxed. This is in agreement with P07 who found that the AXU clusters deviate from the X-ray luminosity-mass relation but are on the optical luminosity-mass relation.

How unusual is A 315 then? Rykoff et al. (2008) found a scatter in luminosity at fixed mass of $\sigma_{\ln L_{X} \mid M}=0.4$, much higher than the scatter in the binned relation shown in Fig. 3. The X-ray luminosity of A 315 given its mass estimate would imply that the cluster deviates by $6 \sigma$ from the $L_{X}$-mass relation, but the uncertainty in the A 315 mass estimates is rather high. However, the kinematical and weak-lensing mass estimates are based on different physical principles and agree well with each other. A weighted average of the two measurements gives a best estimate of $\bar{M}_{200}=\left(2.85_{-0.51-0.45}^{+0.79+0.57}\right) \times 10^{14} h^{-1} M_{\odot}$, so that the cluster deviates by $2 \sigma$ from the $L_{X}$-mass relation along the mass axis. In all cases, A 315 appears to be an unusual cluster compared to the population of X-ray selected clusters that define the $L_{X}$-mass relation.

Weak-lensing and optical-mass estimators are both affected by projections along the line-of-sight (LOS). An extendend structure such as a filament along the LOS or the superposition of smaller groups, would increase both mass estimates. The Xray luminosity, which depends on the square of the density of the intra-cluster medium, would not be enhanced very much in this scenario. While with present data be used to exclude such scenarios, nothing in the data suggests that this is a configuration that we observe. A superposition of groups should produce kinematical substructure. With the few redshifts available from the SDSS, this cannot be decisively excluded but we found no evidence of substructure in Sect. 4. A filament is a low density environment and as such is dominated by blue late-type galaxies (Braglia et al. 2007). We found that 12 of the 14 galaxies inside $r_{200}$ brighter than $L^{*}$ are on the cluster red-sequence. This shows that A 315 is dominated by early-type galaxies as one would expect for a high-density environment such as a cluster.

Studying A 315 and similar X-ray underluminous galaxy clusters is important to understanding the nature of the large scatter in the cluster X-ray luminosity-mass relation. If intrinsic, as supported by our weak-lensing mass measurement of A 315, this scatter implies that X-ray selected cluster samples are incomplete samples in terms of mass. An analysis of the stacked velocity histograms of all RASS-SDSS clusters in P07 showed a leptokurtic distribution, indicative of systems still in formation (Wojtak et al. 2005). In such a system still in formation, the intracluster gas itself may not yet have reached its final temperature.

This scenario could be tested with further spectroscopic and X-ray observations. Data from the SDSS do not provide enough spectra of cluster members to show deviations from Gaussianity in the velocity histogram of individual galaxies. A spectroscopic sample of several hundred cluster galaxies, which can easily be obtained with modern spectrographs, could be used to confirm the leptokurtic velocity distribution for an individual cluster and identify infalling substructure. Similarly, deeper X-ray observations than available in the RASS would allow us to measure the density distribution of the intra-cluster gas. An improved temperature measurement due to higher quality (background) statistics and a more self-consistent X-ray analysis that does not rely on other methods to determine the truncations, could significantly reduce the error in the X-ray luminosity and provide a more definite answer to the question of how unusual Abell 315 really is.

On the theoretical side, SPH simulations of galaxy clusters could be used to generate mock X-ray observations, and semianalytic models of galaxy formation could be used to populate $\mathrm{N}$-body halos with galaxies. The combination of these two methods could provide further insight into the observable properties of galaxy clusters that are not yet fully virialized.

Acknowledgements. We are grateful to Lance Miller and Tom Kitching for providing their lensfit source code to us. Y. Y. Z. acknowledges support by the DFG through Emmy Noether Research Grant RE 1462/2 and by the German BMBF through the Verbundforschung under grant No. 50 OR 0601.

\section{References}

Abell, G. O., Corwin, Jr., H. G., \& Olowin, R. P. 1989, ApJS, 70, 1 Adami, C., Mazure, A., Biviano, A., Katgert, P., \& Rhee, G. 1998, A\&A, 331, 493 
Adelman-McCarthy, J. K., Agüeros, M. A., Allam, S. S., et al. 2007, ApJS, 172, 634

Baade, D., Meisenheimer, K., Iwert, O., et al. 1999, The Messenger, 95, 15

Barkhouse, W. A., Green, P. J., Vikhlinin, A., et al. 2006, ApJ, 645, 955

Beers, T. C., Flynn, K., \& Gebhardt, K. 1990, AJ, 100, 32

Bernstein, G. M., \& Jarvis, M. 2002, AJ, 123, 583

Biviano, A., Murante, G., Borgani, S., et al. 2006, A\&A, 456, 23

Blanton, M. R., \& Roweis, S. 2007, AJ, 133, 734

Böhringer, H., Schuecker, P., Guzzo, L., et al. 2001, A\&A, 369, 826

Bower, R. G., Castander, F. J., Ellis, R. S., Couch, W. J., \& Boehringer, H. 1997, MNRAS, 291, 353

Braglia, F., Pierini, D., \& Böhringer, H. 2007, A\&A, 470, 425

Corless, V. L., \& King, L. J. 2007, MNRAS, 380, 149

Dietrich, J. P., Erben, T., Lamer, G., et al. 2007, A\&A, 470, 821

Dodelson, S. 2004, Phys. Rev. D, 70, 023008

Dolag, K., Bartelmann, M., Perrotta, F., et al. 2004, A\&A, 416, 853

Donahue, M., Scharf, C. A., Mack, J., et al. 2002, ApJ, 569, 689

Dressler, A., \& Shectman, S. A. 1988, AJ, 95, 985

Erben, T., Van Waerbeke, L., Bertin, E., Mellier, Y., \& Schneider, P. 2001, A\&A, 366,717

Erben, T., Schirmer, M., Dietrich, J. P., et al. 2005, Astron. Nachr., 326, 432

Fang, T., Gerke, B. F., Davis, D. S., et al. 2007, ApJ, 660, L27

Gal, R. R. 2006, [arxiv: astro-ph/0601195]

Gal, R. R., de Carvalho, R. R., Lopes, P. A. A., et al. 2003, AJ, 125, 2064

Gilbank, D. G., Bower, R. G., Castander, F. J., \& Ziegler, B. L. 2004, MNRAS, 348,551

Hartmann, D., \& Burton, W. B. 1997, Atlas of Galactic Neutral Hydrogen ed. D. Hartmann, \& W. Butler Burton (Cambridge, UK: Cambridge University Press), 243

Heymans, C., Van Waerbeke, L., Bacon, D., et al. 2006, MNRAS, 368, 1323

Hoekstra, H. 2001, A\&A, 370, 743

Hoekstra, H. 2003, MNRAS, 339, 1155

Ilbert, O., Arnouts, S., McCracken, H. J., et al. 2006, A\&A, 457, 841

Kaiser, N., Squires, G., \& Broadhurst, T. 1995, ApJ, 449, 460

Kalberla, P. M. W., Burton, W. B., Hartmann, D., et al. 2005, A\&A, 440, 775

Kasun, S. F., \& Evrard, A. E. 2005, ApJ, 629, 781

Kim, R. S. J., Kepner, J. V., Postman, M., et al. 2002, AJ, 123, 20
Kitching, T. D., Miller, L., Heymans, C. E., van Waerbeke, L., \& Heavens, A. F. 2008, ArXiv e-prints, 802

Koch, A., Odenkirchen, M., Grebel, E. K., \& Caldwell, J. A. R. 2004, Astron. Nachr., 325, 299

Lubin, L. M., Mulchaey, J. S., \& Postman, M. 2004, ApJ, 601, L9

McNamara, B. R., Vikhlinin, A., Hornstrup, A., et al. 2001, ApJ, 558, 590

Miller, L., Kitching, T. D., Heymans, C., Heavens, A. F., \& van Waerbeke, L. 2007, MNRAS, 382, 315

Morrison, R., \& McCammon, D. 1983, ApJ, 270, 119

Nakajima, R., \& Bernstein, G. 2007, AJ, 133, 1763

Pickles, A. J. 1998, PASP, 110, 863

Popesso, P., Böhringer, H., Brinkmann, J., Voges, W., \& York, D. G. 2004, A\&A, 423, 449

Popesso, P., Böhringer, H., Romaniello, M., \& Voges, W. 2005, A\&A, 433, 415

Popesso, P., Biviano, A., Böhringer, H., \& Romaniello, M. 2007a, A\&A, 461, 397 (P07)

Popesso, P., Biviano, A., Böhringer, H., \& Romaniello, M. 2007b, A\&A, 464, 451

Press, W. H., Teukolsky, S. A., Vetterling, W. T., \& Flannery, B. P. 1992, Numerical recipes in $\mathrm{C}$., The art of scientific computing (Cambridge: University Press), 2nd edn.

Raymond, J. C., \& Smith, B. W. 1977, ApJS, 35, 419

Reiprich, T. H., \& Böhringer, H. 2002, ApJ, 567, 716

Rykoff, E. S., Evrard, A. E., McKay, T. A., et al. 2008, MNRAS, 387, L28

Schlegel, D. J., Finkbeiner, D. P., \& Davis, M. 1998, ApJ, 500, 525

Schneider, P. 1996, MNRAS, 283, 837

Schneider, P., King, L., \& Erben, T. 2000, A\&A, 353, 41

Seitz, S., \& Schneider, P. 2001, A\&A, 374, 740

Shaw, L. D., Weller, J., Ostriker, J. P., \& Bode, P. 2006, ApJ, 646, 815

Staniszewski, Z., Ade, P. A. R., Aird, K. A., et al. 2008, [arXiv:0810.1578]

The, L. S., \& White, S. D. M. 1986, AJ, 92, 1248

Weinberg, N. N., \& Kamionkowski, M. 2002, MNRAS, 337, 1269

Wojtak, R., Łokas, E. L., Gottlöber, S., \& Mamon, G. A. 2005, MNRAS, 361, L1

Zhang, Y.-Y., Böhringer, H., Mellier, Y., Soucail, G., \& Forman, W. 2005, A\&A, 429, 85

Zhang, Y.-Y., Finoguenov, A., Böhringer, H., et al. 2008, A\&A, 482, 451 